\title{
The Development of Brain Network Architecture
}

\author{
Lara M. Wierenga, ${ }^{\prime} *$ Martijn P. van den Heuvel, ${ }^{2}$ \\ Sarai van Dijk,' Yvonne Rijks,' Marcel A. de Reus, ${ }^{2}$ and \\ Sarah Durston' \\ ${ }^{1}$ NICHE Laboratory, Department of Psychiatry, Brain Center Rudolf Magnus, \\ University Medical Center Utrecht, The Netherlands \\ ${ }^{2}$ Department of Psychiatry, Brain Center Rudolf Magnus, \\ University Medical Center Utrecht, The Netherlands
}

\begin{abstract}
Brain connectivity shows protracted development throughout childhood and adolescence, and, as such, the topology of brain networks changes during this period. The complexity of these changes with development is reflected by regional differences in maturation. This study explored age-related changes in network topology and regional developmental patterns during childhood and adolescence. We acquired two sets of Diffusion Weighted Imaging-scans and anatomical T1weighted scans. The first dataset included 85 typically developing individuals (53 males; 32 females), aged between 7 and 23 years and was acquired on a Philips Achieva 1.5 Tesla scanner. A second dataset $(N=38)$ was acquired on a different (but identical) $1.5 \mathrm{~T}$ scanner and was used for independent replication of our results. We reconstructed whole brain networks using tractography. We operationalized fiber tract development as changes in mean diffusivity and radial diffusivity with age. Most fibers showed maturational changes in mean and radial diffusivity values throughout childhood and adolescence, likely reflecting increasing white matter integrity. The largest agerelated changes were observed in association fibers within and between the frontal and parietal lobes. Furthermore, there was a simultaneous age-related decrease in average path length $(P<0.0001)$, increase in node strength $(P<0.0001)$ as well as network clustering $(P=0.001)$, which may reflect fine-tuning of topological organization. These results suggest a sequential maturational model where connections between unimodal regions strengthen in childhood, followed by connections from these unimodal regions to association regions, while adolescence is characterized by the strengthening of connections between association regions within the frontal and parietal cortex. Hum Brain Mapp 37:717-729, 2016. @ 2015 Wiley Periodicals, Inc.
\end{abstract}

Key words: brain development; MRI; DTI; graph theory; brain network

Additional Supporting Information may be found in the online version of this article.

Contract grant sponsor: Netherlands Organisation for Scientific Research (NWO); Contract grant sponsor: VIDI; Contract grant number: 91776384; Contract grant sponsor: VICI; Contract grant number: 453-10-005; Contract grant sponsor: VENI; Contract grant number: 451-12-001; Contract grant sponsor: Brain Center Rudolph Magnus.

*Correspondence to: Lara M. Wierenga, Department of Psychiatry, Brain Center Rudolf Magnus, University Medical Center Utrecht,
HP A01.468, Heidelberglaan 100, 3584 CX, Utrecht, The Netherlands. E-mail: 1.wierenga@umcutrecht.nl

Received for publication 21 May 2015; Revised 6 November 2015; Accepted 9 November 2015.

DOI: $10.1002 / \mathrm{hbm} .23062$

Published online 23 November 2015 in Wiley Online Library (wileyonlinelibrary.com). 


\section{INTRODUCTION}

It is well established that pronounced changes in white matter structure occur early in life [as reviewed in Ball et al., 2014; Dubois et al., 2014; Hagmann et al, 2010; van den Heuvel et al., 2015]. In addition, refinement of brain networks continue throughout childhood and adolescence. This is reflected by increases in total white matter volume, reported in studies using anatomical Magnetic Resonance Imaging (MRI), as well as studies using Diffusion Weighted Imaging (DWI), which show developmental increases in fractional anisotropy (FA) and concurrent decreases in mean diffusion (MD) in white matter bundles. Such relatively late developmental changes are likely to reflect developmental increases in myelination leading to increases in axonal conduction speed [Baumann and Pham-Dinh, 2001], which in turn improve the efficiency of information transfer [van der Knaap et al., 1991]. As all fibers in the brain contribute to network topology, even small changes in white matter structure may have large effects on the properties of the network as a whole. Recent advances in network analysis are now permitting us to investigate such aspects of network topology [Bullmore and Sporns, 2009; He et al., 2006; van den Heuvel and Hulshoff Pol, 2010]. Studies of brain network development in childhood and adolescence have shown increases in network strength and global efficiency [Chen et al., 2013; Dennis et al., 2013; Hagmann et al., 2010; Van den Heuvel et al., 2015]. Furthermore, there is evidence that not all fibers develop at the same rate [Lebel et al., 2008; Tamnes et al., 2010] and may therefore not all contribute equally or simultaneously to global changes in network topology with age.

For example, in newborns, studies have suggested that centrally located white matter develops earlier than in peripheral regions and that occipital white matter develops before that in frontal regions [Gao et al., 2009; Hermoye et al., 2006; Provenzale et al., 2007]. Other studies have shown that the maturation of white matter continues into childhood and adolescence, and that the rates of change may differ per region. For example, the association tracts within frontal and temporal lobes continue to mature for longer than the commissural fibers connecting the hemispheres and the projection fibers connecting subcortical to cortical structures [Dennis et al., 2013; Lebel and Beaulieu, 2011; Tamnes et al., 2010]. Hagmann et al. were one of the first to show that these patterns of white matter maturation contribute to the development of connectivity. They described a maturational pattern with relatively later maturation of long-distance pathways compared with shorter ones [2010]. They showed that this maturational pattern was related to increases in network efficiency, as reflected by a reduction in the number of steps necessary to get from one brain region to another [also reported by Dennis et al., 2013]. Studies of age-related change in functional connectivity support this observation as they show age-related increases in functional connectivity between distant regions, with smaller changes, or even decreases, in short range connectivity [Dosenbach et al., 2010; Fair et al., 2009]. These results still held after agerelated motion effects were removed [Power et al., 2012]. Thus, studies on the developmental patterns of structural white matter are essential for our understanding of the development of network topology, yet they are not all in agreement. Therefore, the first aim of the present study is to describe regional patterns of age-related changes within white matter. Second, we explored developmental patterns in global network topology (node strength, path length, and clustering) and investigate their relation to our regional differences in maturation between fiber tracts.

We used two datasets ( $N=85$ and $N=38$, respectively) of typically developing children and adolescents (aged 7.0-22.9, 61\% males). The datasets were acquired on two 1.5 Tesla Philips Achieva MRI-scanners, allowing us to use the second dataset to replicate findings from the first.

\section{MATERIAL AND METHOD}

The Institutional Review Board (IRB) of the University Medical Center Utrecht in the Netherlands approved this study and its procedures. Written informed consent was obtained for all subjects. For children under 18 years of age, a parent signed for consent. Subjects aged 18 years and older signed for their own consent.

\section{Participants}

Dataset I included 85 typically developing individuals (53 males; 32 females) aged between 7.0 and 22.6 years, with average IQ (see demographic details in Table I). Dataset II included 38 scans in a comparable demographic (23 males; 15 females, aged between 7.4 and 22.9 years). The two datasets were acquired on Philips Achieva 1.5 Tesla MRI scanners, we used the second set for independent replication of our findings. Dataset I and II did not differ in terms of gender distribution, IQ, socio-economic status or hand preference and had a similar age range (see Table I).

We recruited all participants through schools and educational centers in the area. For each subject, a parent participated in a semi-structured interview session with a trained rater to confirm the absence of any psychiatric diagnosis (Diagnostic Interview Schedule for Children [DISC-P]) [Costello et al., 1985]. Subjects older than 18 years old participated in the Mini-International Neuropsychiatric Interview (M.I.N.I) to confirm absence of psychopathological symptoms [Sheehan et al., 1998]. Participants had no psychiatric diagnosis (current or prior), or first-degree relative suffering from a psychiatric illness, no major physical illness of the cardiovascular, the endocrine, the pulmonic or the gastrointestinal system, neurological illness, a history of head trauma with 
unconsciousness, organic brain damage or disease, alcohol or other drug dependence, or full-IQ scale below 75 .

All subjects participated in a neuropsychological assessment (Wechsler Adult Intelligence Scale/Wechsler Adult Intelligence Scale-Third Edition [WAIS/WAIS-III] [Wechsler, 2000]; Wechsler Intelligence Scale for ChildrenRevised/Wechsler Intelligence Scale for Children-Third Edition [WISC-R/WISC-III] [Van Haasen et al., 1986]. Furthermore, participants and their parents filled out a short questionnaire on hand preference and a questionnaire related to major physical or neurological illness. Subjects acclimated to the scanning procedure in a simulated scan session prior to the actual MRI scan [Durston et al., 2009]. An independent clinical neuroradiologist evaluated all MRI-scans and no gross abnormalities were reported for any of the participants.

\section{Image Acquisition and Processing}

\section{Acquisition}

MRI scans were acquired using an identical scan protocol on two identical Philips Achieva 1.5 Tesla scanners, with 6-element SENSE receiver head coils (Philips, Best, The Netherlands). The protocol included a DTI scan and a high-resolution T1-weighted scan (used for anatomical reference). First, we acquired a whole brain T1-weighted three-dimensional fast field echo scan of the whole head (flip angle $=30^{\circ} ; 160-180$ contiguous coronal slices; $1 \times 1$ $\times 1.2 \mathrm{~mm}^{3}$ voxel size; $256 \times 256$ matrix; FoV $=256 \mathrm{~mm}$; $\mathrm{TE}=4.6 \mathrm{~ms} ; \mathrm{TR}=30 \mathrm{~ms}$; and total scan duration $=405-$ $456 \mathrm{~s})$. For white matter fiber tract reconstruction and computation of the MD and RD (radial diffusivity) values, two transverse DTI-sets were acquired, each consisting of 32 diffusion-weighted volumes with different noncollinear diffusion directions with $b$-factor $=1,000 \mathrm{~s} / \mathrm{mm}^{2}$ and 8 diffusion-unweighted volumes with $b$-factor $=0 \mathrm{~s} / \mathrm{mm}^{2}$ (parallel imaging SENSE factor $=2.5$; flip angle $=90^{\circ} ; 60$ slices; $1.875 \times 1.875 \times 2.5 \mathrm{~mm}^{3}$ voxel size; no slice gap; $96 \times 96$ acquisition matrix; reconstruction matrix $128 \times$ 128; $\mathrm{FoV}=240 \mathrm{~mm}$; $\mathrm{TE}=88 \mathrm{~ms}$; $\mathrm{TR}=9822 \mathrm{~ms}$; no cardiac gating; and total scan duration $=296 \mathrm{~s}$ ).

\section{DTI preprocessing and fiber track reconstruction}

The DTI preprocessing pipeline has been described in detail elsewhere [van den Heuvel et al., 2010]. In short, it involved the following steps: First, both DWI sets were realigned and corrected for common gradient-induced distortions. Second, diffusion images were realigned to the $b=0$ image [Andersson and Skare, 2002]. Third, we fitted a diffusion profile within each voxel using the two sets of 32 weighted images and the average $b=0$ image from each subject. From the resulting tensor, the main diffusion direction in each voxel was selected as the principal eigenvector resulting from the eigenvalue decomposition of the fitted tensor, marking the preferred diffusion direction in 
each voxel. For each voxel, the FA, indicating the level of anisotropic diffusion, MD and RD values were computed [Basser and Pierpaoli, 1996; Beaulieu and Allen, 1994]. Fourth, using the extracted information on the preferred diffusion direction within each voxel in the brain mask, the white matter tracts of the whole brain network were reconstructed using the deterministic fiber tracking, based on the FACT algorithm (fiber assignment by continuous tracking) [Mori and van Zijl, 2002; Mori et al., 2001]. This enabled the reconstruction of white matter tracts [van den Heuvel et al., 2009, 2011]. Fibers were reconstructed by starting eight seeds in each voxel and following the main diffusion direction of each voxel (selected as the principal eigenvector) until the fiber tract entered a voxel with a low level of diffusion preference $(\mathrm{FA}<0.1)$, made an unexpected sharp angular turn (angle $>45^{\circ}$ ), or left the brain mask. All reconstructed fibers included a minimum number of five streamlines. One measure of white matter connectivity strength is the level of myelination, as higher levels of myelination have been linked to higher transportation speeds along axonal connections [Wolff and Balaban, 1994]. MD and RD have been associated with myelination [Schmierer et al., 2007]. Therefore, we labeled the strength of each reconstructed fiber with its corresponding $\mathrm{MD}$ and $\mathrm{RD}$ value by assigning each point of the fiber tract the MD and RD values of the voxels along the three-dimensional (3D) path of the fiber [see Hagmann et al., 2010; van den Heuvel et al., 2008, 2009]. The mean MD and RD of each fiber tract were our measures of interest. One of the greatest challenges in DWI is partial volume averaging between tissue types and characteristics, for example, at crossing fibers. This is particularly challenging for the interpretation of FA changes near crossing fibers: FA is particularly sensitive to (age-related) changes in white matter, yet not very specific for the type of microstructural change. Therefore, changes in fiber integrity near crossing fibers may cause both increases and decreases in FA [Alexander et al., 2007; Hagmann et al., 2010; van den Heuvel et al., 2010]. In our data, we found no significant net effect of age on mean FA, due to these increases and decreases that we did observe (see Supporting Information Figure 1 and 2). For this reason, we chose $\mathrm{MD}$ and $\mathrm{RD}$ as markers of white matter development and not FA, similar to the methods proposed by Hagmann et al. [2010].

\section{TI preprocessing}

Within each individual dataset, we used the T1 images for anatomical reference and for the selection of brain network nodes. Tissue classification and anatomical labeling was performed on the basis of the T1-weighted MR image using the well-validated and well-documented Freesurfer v5.1.0 software (http://surfer.nmr.mgh.harvard. edu/). Technical details of the automated reconstruction scheme are described elsewhere [e.g., Dale et al., 1999; Fischl et al., 1999]. Automatic segmentation of subcortical structures (thalamus, pallidum, caudate, putamen, accumbens, hippocampus, amygdala) and parcellation of the reconstructed cortical surface into 68 distinct brain regions was performed using the Desikan-Killiany atlas [Desikan et al., 2006]. Visual data inspection was performed by two independent raters ( $\mathrm{LW}, \mathrm{SvD})$. The nodes of the individual brain networks were therefore represented by a total of 82 brain regions.

\section{Reconstruction of Unweighted and Weighted Graphs}

Each individual's DTI connectivity dataset was represented as a graph, with 82 brain regions defined as the nodes of the graph and the edges between nodes $i$ and $j$ (i.e., brain region $\mathrm{i}$ and region $\mathrm{j}$ ) reflecting a reconstructed white matter tract. For the un-weighted graphs, fibers that touched both region $i$ and $j$ were selected in the total collection of reconstructed tracts, resulting in a undirected binary matrix $\mathrm{G}$ for each individual representing all connections within the brain [van den Heuvel et al., 2008, 2009]. Next, MD- and RD-weighted graphs were created by defining the connection strength of each tract as the average level of MD or RD over the whole fiber (inversely) reflecting the degree of myelination and/or axonal density [Alexander et al., 2007; Yoshida et al., 2013]. This resulted in the undirected weighted connectivity matrix M. As such, the level of MD and RD expressed the strength of each connection.

To effectively reduce the occurrence of falsely positive and falsely negative traced connections, networks were thresholded, retaining only those connections that were present in $60 \%$ of all subjects [de Reus and van den Heuvel, 2013]. In addition, we computed the graph measures at multiple threshold levels, to address the robustness of our findings. Supporting Information Figure 3 shows a connection density matrix of the two datasets, where the number of subjects that share a connection is expressed as a percentage.

\section{Network Metrics}

For graph theoretical analysis, we used the Brain Connectivity Toolbox [Rubinov and Sporns, 2010]. The inverse $\mathrm{MD}$ and $\mathrm{RD}$ values of each edge in matrix $\mathrm{M}$ were used to compute the following measures: average node strength as the average sum of weights of connections to each node; path length as the average minimum number of weighted edges to be traversed to get from one node to another in matrix M [Watts and Strogatz, 1998]; and finally, mean clustering coefficient as the ratio of the number of connections between direct neighbors of a node and the total number of possible connections between these neighbors [Watts and Strogatz, 1998]. 

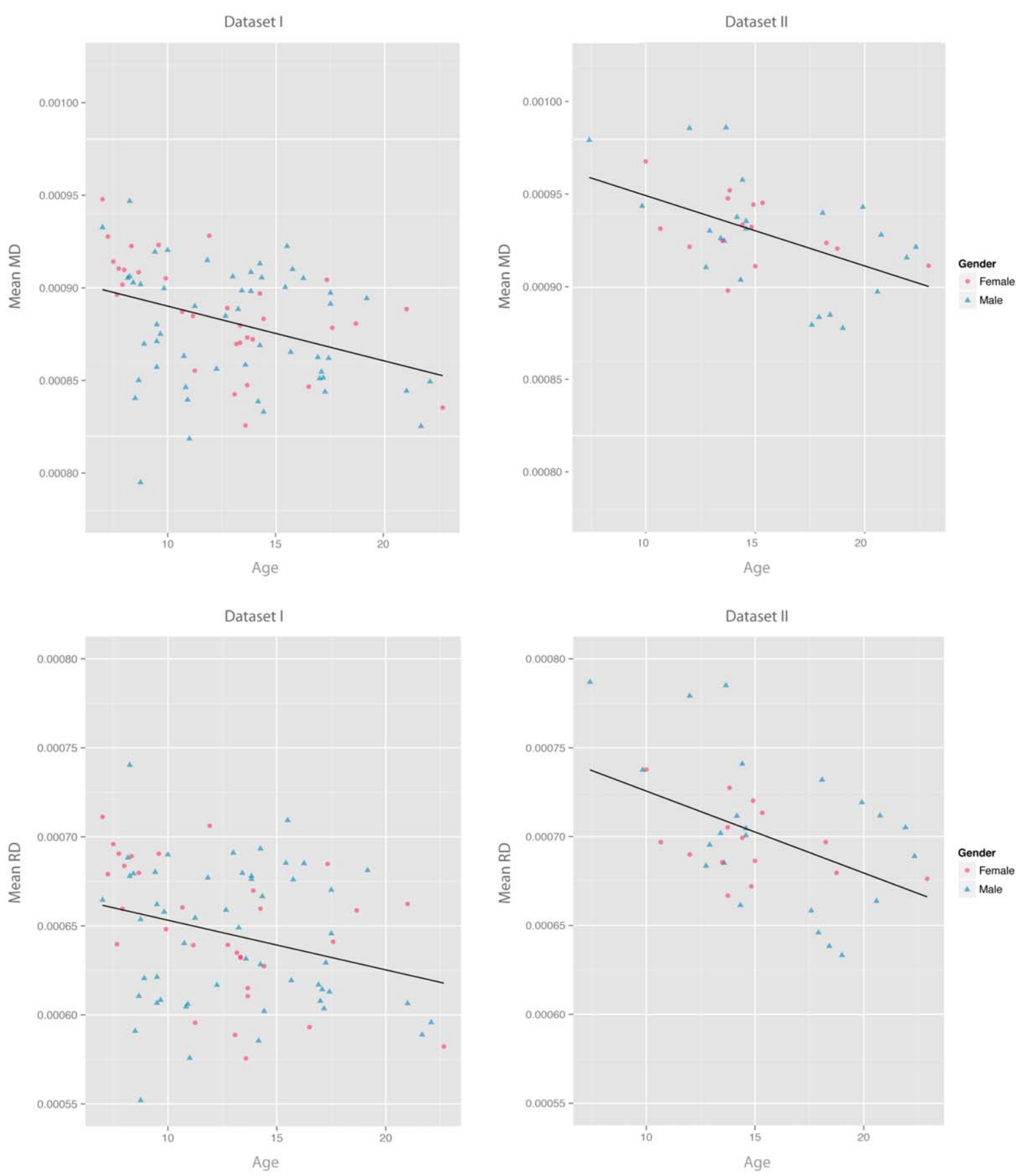

Figure I.

Mean MD and RD decreases with age. Mean MD and RD were calculated per subject by averaging $M D$ and $R D$ values for each connection in the weighted matrix M. Males are shown in blue, females in pink. [Color figure can be viewed in the online issue, which is available at wileyonline library.com.]

\section{Statistical Analyses}

To analyze age-related changes in the number of connections and mean MD and mean RD, Pearson correlations were calculated. The number of connections in each undirected binary matrix $G$ for each subject was correlated with age. Next, mean MD and mean RD were calculated per subject over all connections in each weighted connectivity matrix $\mathrm{M}$ and correlated with age. 


\section{Mean Diffusivity}

Dataset I

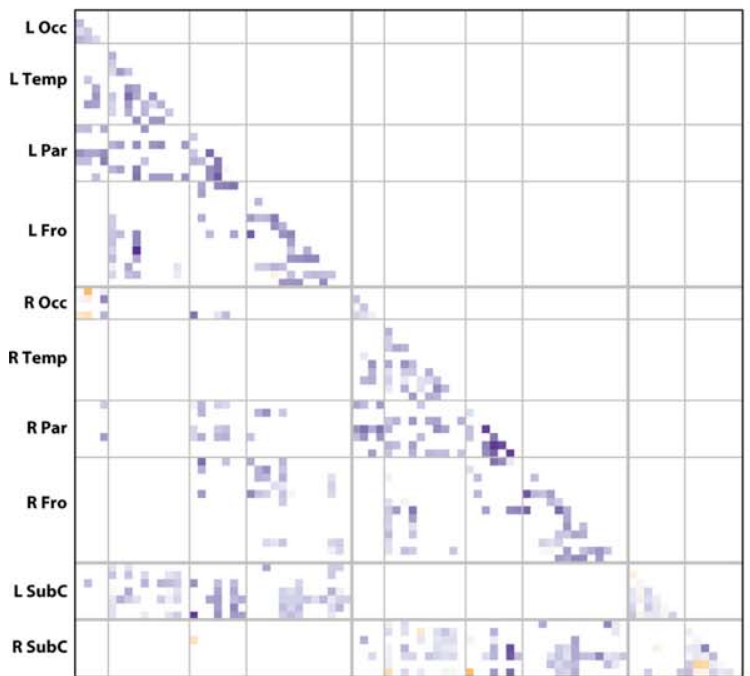

Dataset II

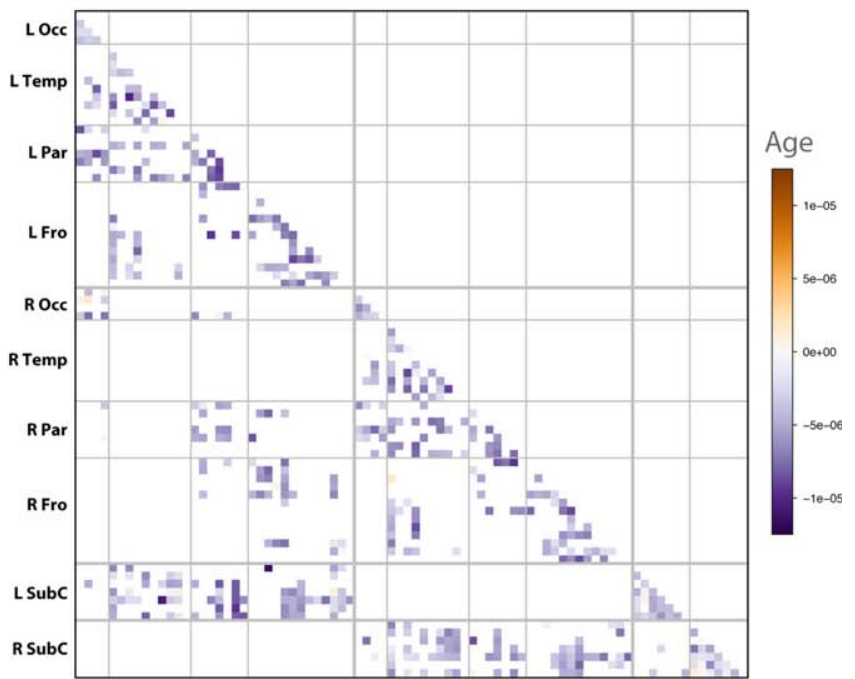

Radial Diffusivity

Dataset I

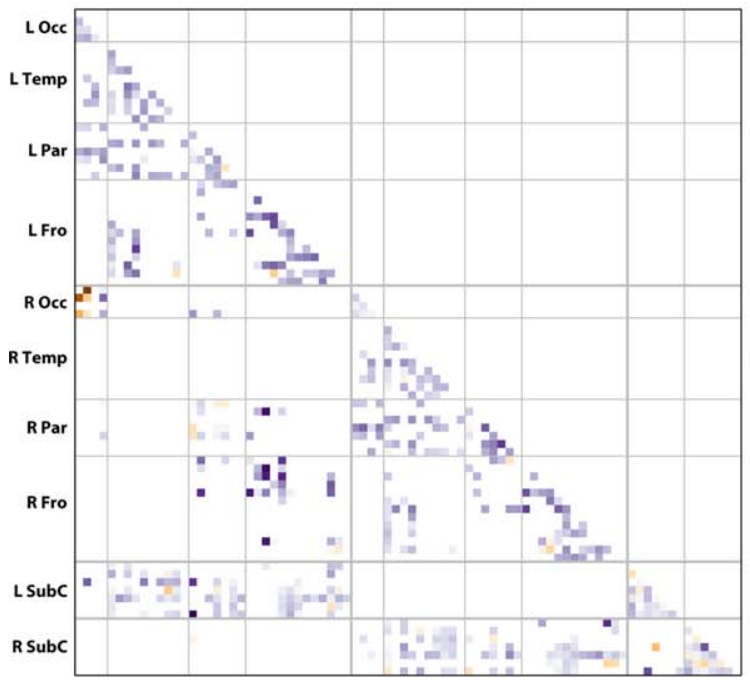

Dataset II

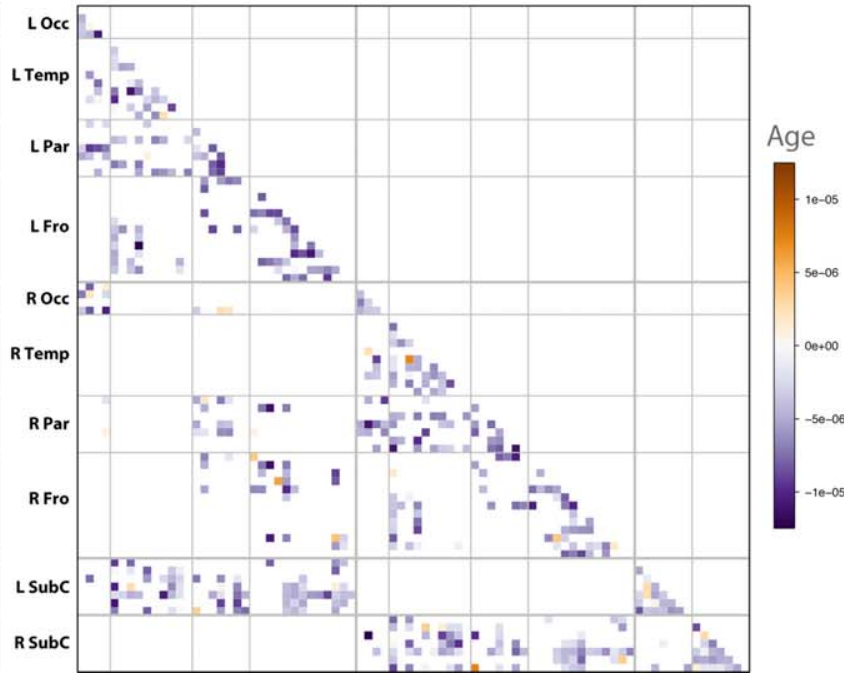

Figure 2.

Age-related changes in MD and RD for the connections in the weighted matrix $M$. Changes are estimated as $\beta_{\text {age }}$ for all connections that were present in at least $60 \%$ of the participants. We found widespread decreases in MD and RD with age (shown in purple) and only limited increases (shown in orange). $R$ SubC $=$ right subcortical structures, $\mathrm{L}$ SubC $=$ left subcortical

Furthermore, we used a linear model procedure to examine the relation between age and $\mathrm{MD} / \mathrm{RD}$ values on each single connection in the weighted matrix $\mathrm{M}$ and network metrics. structures, $\mathrm{R}$ Fro = right frontal lobe, $\mathrm{R}$ Par $=$ right parietal lobe, $r$ Temp $=$ right temporal lobe, $\mathrm{R}$ Occ $=$ right occipital lobe, $\mathrm{L}$ Fro $=$ left frontal lobe, $L$ Par $=$ left parietal lobe, $L$ Temp $=$ left temporal lobe and $\mathrm{L}$ Occ $=$ left occipital lobe. [Color figure can be viewed in the online issue, which is available at wileyonline library.com.]

Note that only connections that were present in more than $60 \%$ of the subjects were taken into account. Each dependent measure of the $i^{\text {th }}$ individual was modeled as follows: 

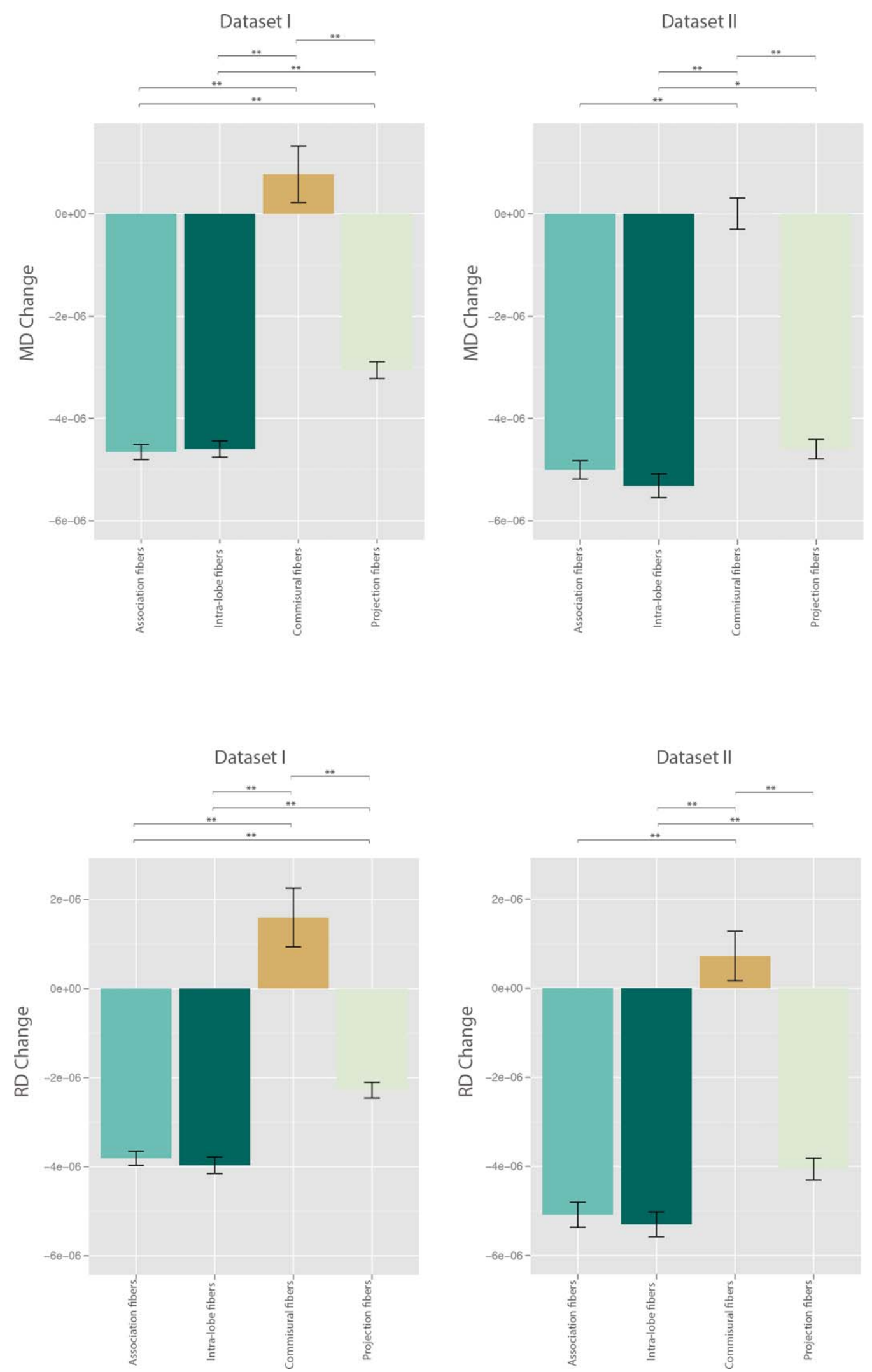

Figure 3.

Age-related changes are greatest for association and intra-lobe ciation, intralobe, commissural and projection fibers. fibers. The $\mathrm{Y}$-axis shows age-related change (estimated as $\beta_{\text {age }}$ ) $*=P<0.05, * *=P<0.01$. [Color figure can be viewed in the in MD and RD per connection for each fiber class (x-axis): asso- online issue, which is available at wileyonlinelibrary.com.] 

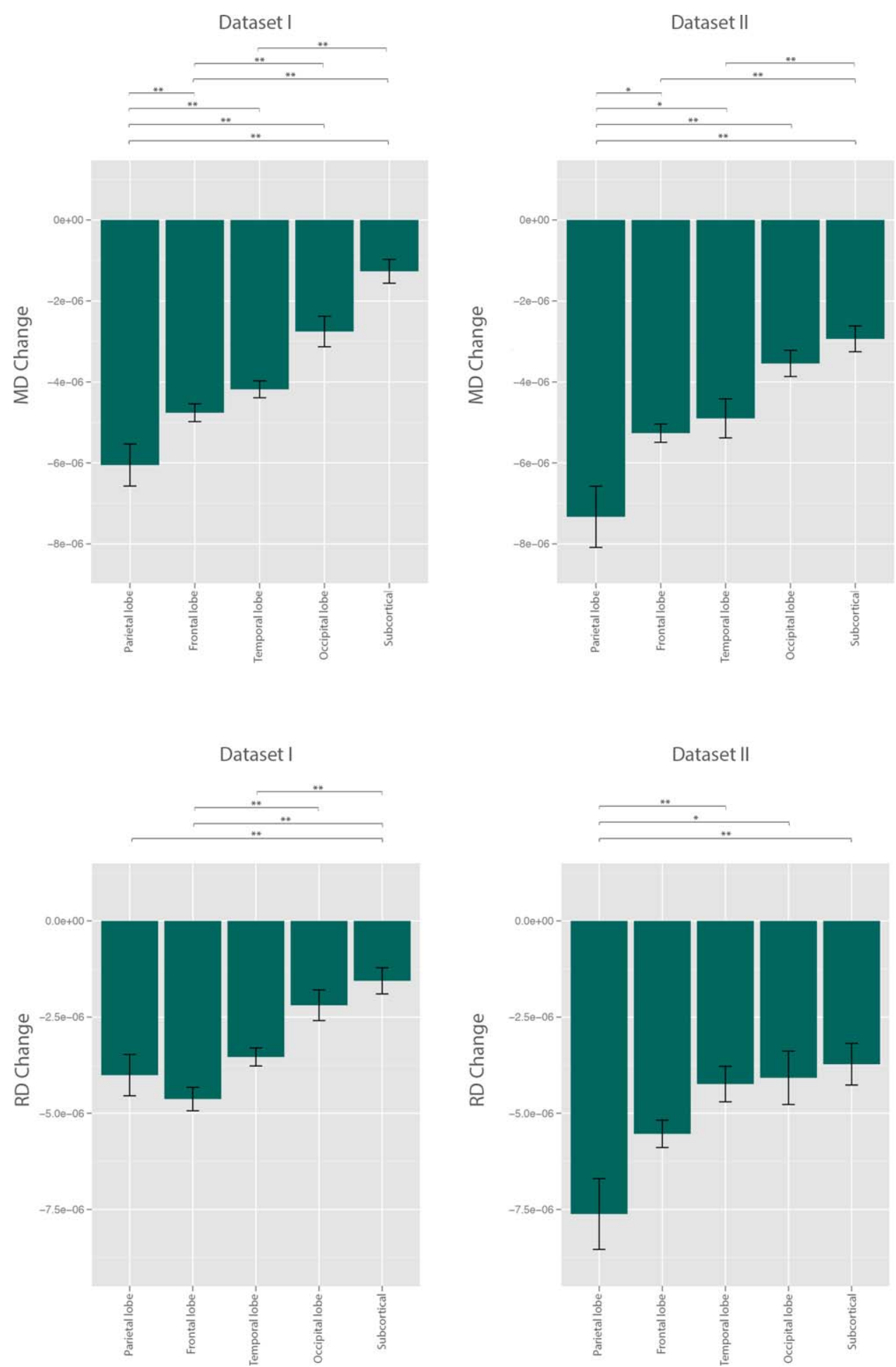

Figure 4.

Age-related changes within lobes are greatest for parietal and fibers connecting subcortical structures., $\quad *=P<0.05$, frontal lobes. The Y-axis shows age-related changes (estimated as $\left.\beta_{\text {age }}\right)$ in MD and RD for intralobe fibers per lobe (x-axis); $* *=P<0.01$. [Color figure can be viewed in the online issue, parietal lobe, frontal lobe, temporal lobe, occipital lobe, and 

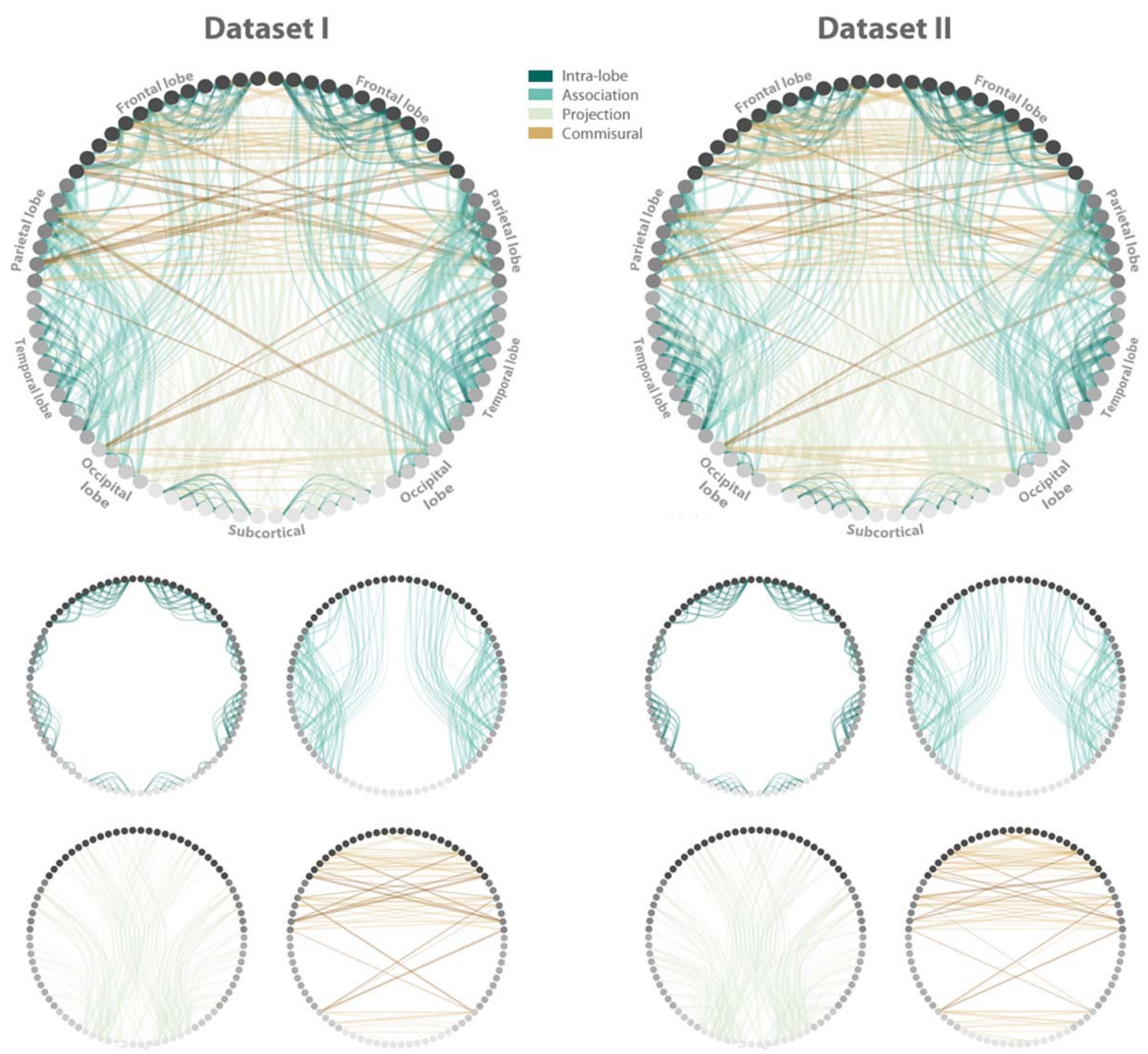

Figure 5.

Age-related changes in MD for each connection in the weighted matrix M. A link between two structures on the circle indicates the presence of a connection between them. Thicker lines reflect a greater rate of developmental change and therefore indicate protracted development. Color coding indicates the different fiber classes. [Color figure can be viewed in the online issue, which is available at wileyonlinelibrary.com.]

$$
\begin{aligned}
& \text { Measurement }{ }_{i}=\text { Intercept }+\beta_{\text {age }} * \text { Age }_{i} \\
& +\beta_{\text {gender }} * \text { Gender }_{i}+\beta_{\mathrm{ICV}} * \mathrm{ICV}_{i}+e_{i}
\end{aligned}
$$

with $e_{i}$ being the normally distributed residual error and intercept, age, gender, and intracranial volume (ICV) being fixed effects [Yan et al., 2011]. (The possible presence of quadratic age-related curves was investigated by adding an age*age term to the regression model, but this did not improve the model fit). As the focus was on age-related

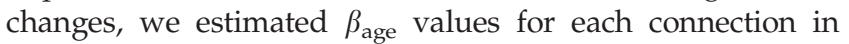
the weighted connectivity matrix $M$. Next, $\beta_{\text {age }}$ values for each connection were subdivided into the four lobes and four fiber categories: intralobe, association (within hemisphere connections between the different lobes), commissural (connecting both hemispheres), and projection fibers (connecting subcortical with cortical structures). Agerelated changes were compared between fiber categories using ANOVA and post hoc t-tests (Bonferroni corrected).

\section{RESULTS}

\section{Age-Related Changes in Unweighted Graphs G}

No changes with age were found in the total amount of (binary) connections in the individual unweighted graphs 
- Wierenga et al. •

TABLE II. The effect of age on network metrices

\begin{tabular}{|c|c|c|c|c|c|c|c|c|}
\hline & \multicolumn{4}{|c|}{ Inverse MD } & \multicolumn{4}{|c|}{ Inverse RD } \\
\hline & $\beta_{\text {age }}$ & (s.e.) & $P$ & & $\beta_{\text {age }}$ & (s.e.) & $P$ & \\
\hline \multicolumn{9}{|l|}{ Dataset I } \\
\hline Node strength & 53.506 & (14.075) & $<0.0001$ & $* *$ & 79.092 & (27.197) & 0.005 & $* *$ \\
\hline Path length & $-1.113 \times 10^{-5}$ & $\left(2.253 \times 10^{-6}\right)$ & $<0.0001$ & $* *$ & $-8.052 \times 10^{-6}$ & $\left(2.387 \times 10^{-6}\right)$ & 0.001 & $* *$ \\
\hline Clustering & 2.389 & $(0.689)$ & 0.001 & $* *$ & 3.908 & (1.320) & 0.004 & $* *$ \\
\hline \multicolumn{9}{|l|}{ Dataset II } \\
\hline Node strength & 39.435 & (18.133) & 0.037 & * & 86.689 & $(29.715)$ & 0.006 & $* *$ \\
\hline Path length & $-1.112 \times 10^{-5}$ & $\left(2.866 \times 10^{-6}\right)$ & $<0.0001$ & $* *$ & $-7.866 \times 10^{-6}$ & $\left(3.264 \times 10^{-6}\right)$ & 0.022 & $*$ \\
\hline Clustering & 1.288 & $(0.934)$ & 0.178 & n.s. & 2.862 & $(1.706)$ & 0.103 & n.s. \\
\hline
\end{tabular}

s.e., standard error; n.s., not significant; ${ }^{*} P<0.05 ;{ }^{* *} P<0.01$.

(dataset I: $R=-0.17, P=0.11$, replication dataset II: $R=-0.04, P=0.81$ ), supporting earlier observations of the majority of tracts being present in the early developing brain [Hagmann et al., 2010; van den Heuvel et al., 2015].

\section{Age-Related Changes in MD- and RD-Weighted Graphs M}

Mean MD decreased with age (dataset I: $R=-0.37$, $P<0.001$, replication dataset II: $R=-0.52, P<0.001$; Fig. 1 ). Mean RD also decreased with age (dataset $\mathrm{I}: R=-0.28$, $P<0.01$, replication dataset II: $R=-0.47, P<0.01$; Fig. 1 ). We found linear decreases in MD and RD for almost all connections, reflected by negative $\beta_{\text {age }}$ values (Fig. 2). ANOVA showed that there was variation in the rate of age-related change between fiber groups for both MD (dataset I: $\mathrm{F}(3,414)=42.63, P<0.001$, replication dataset II: $\mathrm{F}(3,412)=17.09, \quad P<0.001$ ) and RD (dataset I: $\mathrm{F}(3,427)=56.29, P<0.001$, replication dataset II: $\mathrm{F}(3,420)=$ 20.72, $P<0.001$ ). The largest changes (i.e., global decreases in MD and RD) were found for association and intralobe fibers (Fig. 3). Post hoc t-tests (Bonferroni corrected) confirmed that the rate of change in these fibers was larger as compared to the class of commissural and projection fibers $(P<0.001)$. Furthermore, there was variation between lobes in both MD-weighted graphs (dataset I: $F(4,179)=$ 33.78, $P<0.001$, replication dataset II: $\mathrm{F}(4,178)=13.64$, $P<0.001$ ) and RD-weighted graphs (dataset I: $\mathrm{F}(4,179)=$ 13.43, $P<0.001$, replication dataset II: $\mathrm{F}(4,179)=6.23$, $P<0.002$, Fig. 4). Here, we found the greatest age-related decreases in parietal lobe, followed by frontal lobe, temporal lobe, occipital lobe and finally subcortical structures (Bonferroni corrected, see Figs. 4 and 5).

\section{Age-Related Changes in Network Metrics}

There were no effects of ICV or gender on any of the network measures therefore these terms were dropped from the model. Age-related changes in inverse MD- and inverse RD-weighted graphs are given in Table II. Average node strength increased with age for both MD and RD- weighted graphs in dataset I (MD: $\beta_{\text {age }}=53.506$, $t(80)=3.802, P<0.0001, \quad R D: \quad \beta_{\text {age }}=79.092, \quad t(80)=2.908$, $P=0.005)$ as well as in replication dataset II (MD: $\beta_{\text {age }}=39.435, t(32)=2.175, \quad P=0.037, \quad R D: \quad \beta_{\text {age }}=86.689$, $t(32)=2.917, P=0.006)$. Average path length significantly decreased with age in dataset I (MD: $\beta_{\text {age }}=-1.113^{*} 10^{-5}$, $t(80)=5.011, P<0.0001$, RD: $\quad \beta_{\text {age }}=-8.052 * 10^{-6}, t(80)=$ 3.372, $P=0.001)$, this result was replicated in replication dataset II (MD: $\beta_{\text {age }}=-1.112^{*} 10^{-5}, t(32)=3.904, P<.0001$, RD: $\left.\beta_{\text {age }}=-7.866^{*} 10^{-6}, t(32)=2.409, P=.022\right)$. Mean clustering coefficient increased with age for MD-weighted graphs in dataset I (MD: $\beta_{\text {age }}=2.389, t(80)=3.467$, $P=0.001$, RD: $\left.\beta_{\text {age }}=3.908, t(80)=2.961, P=0.004\right)$ but did not reach significance in replication dataset II. To test the stability of the network reconstruction we tested these age effects over different group threshold levels (20, 40, 60, and $80 \%$ ). We observed that the age effects were not significantly different between group threshold levels for node strength $(P=0.965)$, average path length $(P=0.923)$, or clustering $(P=0.992)$.

\section{DISCUSSION}

In this study, we show maturation of brain connectivity throughout adolescence. The developmental changes appear not to be uniform, but rather show regional heterogeneity, with greater strengthening of association and intra-lobe connections than commissural and projection fibers. Furthermore, in the age range of 7 to 23, relatively strong developmental changes in intra-lobe connections within the frontal and parietal lobes compared to changes in temporal and occipital lobes and between subcortical structures were observed. Increased node strength and global clustering in addition to decreased average path length accompanied these changes in connection strength.

Our findings on network topology are in agreement with the findings of Dennis et al. [2013] and Hagmann et al. [2010], who also reported a decrease in overall path length with age and an increase in node strength, respectively. This is in line with previous studies showing maturational increases in efficiency of functional 
communication over the network [Supekar et al., 2009]. We observed that those connections that are potentially involved with information integration in higher order association frontal and parietal areas, show the largest age related change, suggesting that changes in these fiber pathways contribute most to the observed changes in network topology within this age range. We also replicated an increase in clustering with age, as was previously observed by Dennis et al. [2013].

Differences in the rate of age-related changes between fibers could be interpreted as differences in maturational rate: a lower rate of change could be taken to suggest more stable or completed development. Earlier work by Lebel et al. [2008] showed that white matter development levels off in adulthood [2008], with maturational rate slowing. If we adhere to this line of interpretation, our results could be taken to suggest that projection and commissural fibers are the first to mature followed by association and intralobe fibers. Within the intralobe fiber category occipital fibers are the first to mature, followed by temporal fibers, whereas parietal and frontal projections show a more protracted development. This is in keeping with the posterior to anterior pattern of white matter development that has been reported in post-mortem studies [Benes, 1989; Yakovlev and Lecours, 1967] and in anatomical MRIstudies of cortical brain development [Gogtay et al., 2004; Sowell et al., 2001; Wierenga et al., 2013].

Our results complement earlier reports of relatively late maturation of short association-fibers and intralobar connections between parietal and frontal regions throughout adolescence and adulthood [Chen et al., 2013; Lebel and Beaulieu, 2011; Westlye et al., 2010]. Taken together with these earlier observations, our findings suggest a sequential developmental pattern, with early strengthening of connections between unimodal regions, followed by the maturation of connections from these regions to association areas, and finally by maturation of connections between association regions.

There are several limitations to this study. First the male to female ratio was uneven (5:3). Given the relatively small number of female subjects, we may have had limited power to detect gender differences. We did include gender in our regression model, as studies have reported differences in brain development between males and females [see e.g. Lenroot et al., 2007; Ruigrok et al., 2014]. Less then $2 \%$ of the connections showed significant effects of gender. Furthermore, we included ICV to account for the effect of brain size, which improved model fit for $17 \%$ of the connections. Second, we could not replicate nonlinear age-related changes in white matter tracts, as have been previously observed [Lebel et al., 2008]. We may not have been able to detect such non-linear changes due to limited power or the age-range included. Sensitivity to such changes could be improved by including longitudinal data.

In sum, our data support the hypothesis that age-related strengthening of white matter fibers is not homogenous throughout the brain during childhood and adolescence. Rather, we find that development seems to be characterized by strengthening of relatively short association fibers, particularly those within and between the frontal and parietal lobes. This suggests that changes in these connections contribute most to changes in network topology during the same developmental time period.

\section{ACKNOWLEDGMENTS}

The authors thank all subjects and their parents for participating in this study. They further wish to thank Sara Ambrosino for her contribution to quality monitoring, Juliette Weusten, Lizanne Schweren, Fenny S. Zwart, and Sanne Veerhoek for their help in subject recruitment and acquisition of MRI scans. All authors declare no competing financial interests.

\section{REFERENCES}

Alexander AL, Lee JE, Lazar M, Field AS (2007): Diffusion tensor imaging of the brain. Neurotherapeutics 4:316-329.

Andersson J, Skare S (2002): A Model-Based Method for Retrospective Correction of Geometric Distortions in DiffusionWeighted EPI. Neuroimage 16:177-199.

Ball G, Aljabar P, Zebari S, Tusor N, Arichi T, Merchant N, Robinson EC, Ogundipe E, Rueckert D, Edwards AD, Cousell SJ (2014): Rich-club organization of the newborn human brain. Proc Natl Acad Sci U S A 111:7456-7461.

Basser PJ, Pierpaoli C (1996): Microstructural and physiological features of tissues elucidated by quantitative-diffusion-tensor MRI. J Magn Reson B 111:209-219.

Baumann N, Pham-Dinh D (2001): Biology of oligodendrocyte and myelin in the mammalian central nervous system. Physiol Rev 81:871-927.

Beaulieu C, Allen PS (1994): Determinants of anisotropic water diffusion in nerves. Magn Reson Med 31:394-400.

Benes FM (1989): Myelination of cortical-hippocampal relays during late adolescence. Schizophr Bull 15:585

Bullmore E, Sporns O (2009): Complex brain networks: graph theoretical analysis of structural and functional systems. Nat Rev Neurosci 10:186-198.

Chen Z, Liu M, Gross DW, Beaulieu C (2013): Graph theoretical analysis of developmental patterns of the white matter network. Front Human Neurosci 7:716

Costello EJ, Edelbrock CS, Costello AJ (1985): Validity of the NIMH Diagnostic Interview Schedule for Children: A comparison between psychiatric and pediatric referrals. J Abnorm Child Psychol 13:579-595.

Dale A, Fischl B, Sereno MI (1999): Cortical Surface-Based Analysis: I. Segmentation and Surface Reconstruction. Neuroimage 9: 179-194.

de Reus MA, van den Heuvel MP (2013): Estimating false positives and negatives in brain networks. Neuroimage 70:402-409.

Dennis EL, Jahanshad N, McMahon KL, de Zubicaray GI, Martin NG, Hickie IB, Toga AW, Wright MJ, Thompson PM (2013): Development of brain structural connectivity between ages 12 and 30: A 4Tesla diffusion imaging study in 439 adolescents and adults. Neuroimage, 64:671-684. 
Desikan RS, Ségonne F, Fischl B, Quinn BT, Dickerson BC, Blacker D, Buckner RL, Dale AM, Maguire RP, Hyman BT, Albert MS, Killiany RJ (2006): An automated labeling system for subdividing the human cerebral cortex on MRI scans into gyral based regions of interest. Neuroimage, 31:968-980. doi:10.1016/ j.neuroimage.2006.01.021

Dosenbach NUF, Nardos B, Cohen AL, Fair DA, Power JD, Church JA, Nelson SM, Wig GS, Vogel AC, Lessov-Schlaggar CN, Barnes KA, Dubis JW, Feczko E, Coalson RS, Pruett JR, Barch DM, Petersen SE, Schlaggar BL (2010): Prediction of individual brain maturity using fMRI. Science 329:1358-1361.

Dubois J, Dehaene-Lambertz G, Kulikova S, Poupon C, Hüppi PS, Hertz-Pannier L (2014): The early development of brain white matter: A review of imaging studies in fetuses, newborns and infants. Neuroscience 276:48-71.

Durston S, Nederveen H, van Dijk S, van Belle J, de Zeeuw P, Langen M, van Dijk A (2009): Magnetic Resonance Simulation is Effective in Reducing Anxiety Related to Magnetic Resonance Scanning in Children. J Am Acad Child Adolesc Psychiatry 48:206-207.

Fair DA, Cohen AL, Power JD, Dosenbach NUF, Church JA, Miezin FM, Schlaggar BL, Petersen SE (2009): Functional brain networks develop from a "local to distributed" organization. PLoS Comput Biol 5:e1000381

Fischl B, Sereno MI, Dale A (1999): Cortical Surface-Based Analysis: II: Inflation, Flattening, and a Surface-Based Coordinate System. Neuroimage 9:195-207.

Gao W, Lin W, Chen Y, Gerig G, Smith JK, Jewells V, Gilmore JH (2009): Temporal and spatial development of axonal maturation and myelination of white matter in the developing brain. AJNR 30:290-296.

Gogtay N, Giedd J, Lusk L, Hayashi K, Greenstein D, Vaituzis A, Nugent TF, Herman DH, Clasen LS, Toga AW, Rapoport JL, Thompson PM (2004): Dynamic mapping of human cortical development during childhood through early adulthood. Proc Natl Acad Sci U S A 101:8174-8179.

Hagmann P, Sporns O, Madan N, Cammoun L, Pienaar R, Wedeen VJ, Meuli R, Thiran JP, Grant PE (2010): White matter maturation reshapes structural connectivity in the late developing human brain. Proc Natl Acad Sci 107:19067-19072.

He Y, Chen ZJ, Evans AC (2006): Small-World Anatomical Networks in the Human Brain Revealed by Cortical Thickness from MRI. Cerebral Cortex 17:2407-2419.

Hermoye L, Saint-Martin C, Cosnard G, Lee SK, Kim J, Nassogne MC, Menten R, Clapuyt P, Donohue PK, Hua K, Wakana S, Jiang H, van Zijl PCM, Mori S (2006): Pediatric diffusion tensor imaging: Normal database and observation of the white matter maturation in early childhood. Neuroimage 29:493-504.

Lebel C, Beaulieu C (2011): Longitudinal Development of Human Brain Wiring Continues from Childhood into Adulthood. J Neurosci 31:10937-10947.

Lebel C, Walker L, Leemans A, Phillips L, Beaulieu C (2008): Microstructural maturation of the human brain from childhood to adulthood. Neuroimage 40:1044-1055.

Lenroot RK, Gogtay N, Greenstein DK, Wells EM, Wallace GL, Clasen LS, Blumenthal JD, Lerch J, Zijdenbos AP, Evans AC, Thompson PM, Giedd JN (2007): Sexual dimorphism of brain developmental trajectories during childhood and adolescence. Neuroimage 36:1065-1073.

Mori S, van Zijl PCM (2002): Fiber tracking: Principles and strategies - a technical review. NMR Biomed 15:468-480.
Mori S, Crain BJ, Chacko VP, van Zijl PCM (2001): Three-dimensional tracking of axonal projections in the brain by magnetic resonance imaging. Ann Neurol 45:265-269.

Power JD, Barnes KA, Snyder AZ, Schlager BL, Petersen SA (2012): Spuerious but systematic correlations in functional connectivity MRI networks arise from subject motion. Neuroimage 59:2142-2154.

Provenzale JM, Liang L, DeLong D, White LE (2007): Diffusion tensor imaging assessment of brain white matter maturation during the first postnatal year. Am J Roentgenol 189:476-486.

Rubinov M, Sporns O (2010): Complex network measures of brain connectivity: Uses and interpretations. Neuroimage 52:1059-1069.

Ruigrok ANV, Salimi-Khorshidi G, Lai MC, Baron-Cohen S, Lombardo MV, Tait RJ, Suckling J (2014): A meta-analysis of sex differences in human brain structure. Neurosci Biobehav Rev 39:34-50.

Schmierer K, Wheeler-Kingshott CAM, Boulby PA, Scaravilli F, Altmann DR, Barker GJ, Tofts PS, Miller DH (2007): Diffusion tensor imaging of post mortem multiple sclerosis brain. Neuroimage 35:467-477.

Sheehan DV, Lecrubier Y, Sheehan KH, Amorim P, Janavs J, Weiller E, Hergueta T, Baker R, Dunbar GC (1998): The MiniInternational Neuropsychiatric Interview (M.I.N.I.): The development and validation of a structured diagnostic psychiatric interview for DSM-IV and ICD-10. J Clin Psychiatry 59:22-33; quiz 34-57.

Sowell ER, Thompson PM, Tessner KD, Toga AW (2001): Mapping continued brain growth and gray matter density reduction in dorsal frontal cortex: Inverse relationships during postadolescent brain maturation. J Neurosci 21:8819-8829.

Supekar K, Musen M, Menon V (2009): Development of large-scale functional brain networks in children. PLoS Biol 7:e1000157

Tamnes CK, Ostby Y, Fjell AM, Westlye LT, Due-Tonnessen P, Walhovd KB (2010): Brain Maturation in Adolescence and Young Adulthood: Regional Age-Related Changes in Cortical Thickness and White Matter Volume and Microstructure. Cerebral Cortex 20:534-548.

van den Heuvel MP, Mandl RCW, Kahn RS, Hulshoff Pol HE (2009): Functionally linked resting-state networks reflect the underlying structural connectivity architecture of the human brain. Human Brain Mapp 30:3127-3141.

van den Heuvel MP, Mandl RCW, Stam CJ, Kahn RS, Hulshoff Pol HE (2010): Aberrant frontal and temporal complex network structure in schizophrenia: A graph theoretical analysis. J Neurosci 30:15915-15926.

van den Heuvel MP, Kersbergen KJ, de Reus MA, Keunen K, Kahn RS, Groenendaal de Vries LS, Benders MJNL (2015). The Neonatal Connectome During Preterm Brain Development. Cerebral Cortex, 25:3000-3013.

van der Knaap MS, Valk J, Bakker CJ, Schooneveld M, Faber JA, Willemse J, Gooskens RH (1991): Myelination as an expression of the functional maturity of the brain. Dev Med Child Neurol 33:849-857.

Van Haasen PP, De Bruyn EEJ, Pijl YJ, Poortinga YH, LutjeSpelberg HC, Vander Steene G, Coetsier P, Spoelders-Claes R, Stinissen J (1986): Wechsler Intelligence Scale for ChildrenRevised, Dutch Version.

Westlye LT, Walhovd KB, Dale AM, Bjornerud A, Due-Tonnessen P, Engvig A, Grydeland H, Tamnes CK, Østby Y, Fjell AM (2010): Life-Span Changes of the Human Brain White Matter: Diffusion Tensor Imaging (DTI) and Volumetry. Cerebral Cortex 20:2055-2068. 
Wierenga LM, Langen M, Oranje B, Durston S (2013): Unique developmental trajectories of cortical thickness and surface area. Neuroimage 87:120-126.

Wolff SD, Balaban RS (1994): Magnetization transfer imaging: Practical aspects and clinical applications. Radiology 192: 593-599.

Yakovlev PI, Lecours AR (1967): The myelogenetic cycles of regional maturation of the brain. In: Minkowski A, editor.
Regional Development of the Brain Early in Life. Boston, Massachusetts: Blackwell Scientific Publications. pp 3-70.

Yan C, Gong G, Wang J, Wang D, Liu D, Zhu C, Chen ZJ, Evans A, Zang Y, He Y (2011): Sex- and Brain Size-Related SmallWorld Structural Cortical Networks in Young Adults: A DTI Tractography Study. Cerebral Cortex 21:449-458.

Yoshida S, Oishi K, Faria AV, Mori S (2013): Diffusion tensor imaging of normal brain development. Pediatric Radiol 43:15-27. 